\title{
MHD Stability Analysis with Higher Order Spline Functions
}

\author{
IDA Akihiro, TODOROKI Jiro ${ }^{1 \% *}$ and SANUKI Heiji ${ }^{1)}$ \\ Graduate School of Engineering, Nagoya University, Nagoya 464-8602, Japan

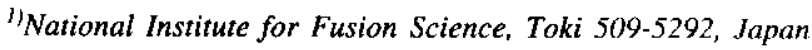

(Received 17 December 1998 / Accepted 22 February 1999)

\begin{abstract}
The eigenvalue problem of the linearized magnetohydrodynamic (MHD) equation is formulated by using higher order spline functions as the base functions of Ritz-Galerkin approximation. When the displacement vector normal to the magnetic surface (in the magnetic surface) is interpolated by B-spline functions of degree $p_{1}$ (degree $p_{2}$ ) which is continuously $c_{1}$-th $\left(c_{2}\right.$-th) differentiable on neighboring finite elements, the sufficient conditions for the good approximation is given by $p_{1} \geq p_{2}+1, c_{1} \leq c_{2}+1,\left(c_{1} \geq 1\right.$, $p_{2} \geq c_{2} \geq 0$ ). The influence of the numerical integration upon the convergence of calculated eigenvalues is discussed.
\end{abstract}

\section{Keywords:}

MHD stability, finite element method, B-spline function, high accuracy, non-compact operator, spectrum pollution, numerical integration

\section{Introduction}

MHD stability is an essential issuc for the magnetic confinement of fusion plasmas. Spectrum analyzing codes of the linearized MHD equation with higher accuracy is required to analyze the localized modes with small growth rate.

The finite element method based on the variational energy principles has been applied to the analysis of MHD spectrum, and a lot of codes have been constructed [1-6], Most of these codes are of second order accuracy, i.e., the numerical errors in the eigenvalues scale as $N^{-2}, N$ being the number of elements in one dimension. Although fourth order accuracy is achieved in NOVA code [7] which does not depend on variational energy principles, by utilizing the third order spline functions, the nonlinear eigenvalue problem is involved. In the present paper, higher order spline functions are used as the base functions of the finite element method which applied to the Lagrangian of the linearized MHD equation.
In the present method, the 2-D (or 3-D) stability problem is reduced to 1-D problem with many variables by using the Fourier expansions with respect to poloidal (and toroidal) angles. Then the finite element method is applied to the minor radius direction. When the displacement vector normal to the magnetic surface is represented by $\boldsymbol{X}$ and the displacement vector in the magnetic surface is represented by $\boldsymbol{Y}$, there appear the differential of $X$ with respect to the minor radius direction, but not the differential of $\boldsymbol{Y}$. The component vectors $\boldsymbol{X}$ and $\boldsymbol{Y}$ belong to the different function spaces. The operator which is defined by the energy integral is not compact; the spectrum pollution can occur. In order to avoid the spectrum pollution most of variational codes employ piecewise linear functions and step functions in hybrid. In this paper, the properties of "good approximation" is defined as the properties which the approximated spectrum should possess, and the sufficient condition for the base functions that "good approximation" is derived is given with a mathematical

*corresponding author's e-mail: todoroki@nifs.ac.jp 
proof.

Most of theorics of the finite element method depend on an assumption that the integral is carried out exactly. However, most of the spectnum analyzing codes utilize the numerical integration for calculating energy integral. Effect of numerical integration on convergence property of eigenvalues is one of the key problems, but only few discussions have so far been made. If the energy function is integrated exactly, the lowest eigenvalue is necessarily approximated from above. In ERATO code [1,2], however, the lowest eigenvalue is approximated from below in most calculations against the ordinary theory. This problem is also discussed in this article.

The bilinear form which defines the linearized MHD operator is introduced in section 2. In section 3, the properties required for the approximated spectrum are defined. The theorem which determines the function spaces of the Ritz-Galerkin approximation is presented in section 4. The outline of the proof of the theorem is given in section 5 (the detail is shown in Appendix II). In section 6, the numerical errors in the eigenvalues are estimated. In section 7 , the influence of the numerical integration upon the convergence of calculated eigenvalues is discussed. The numerical examples in the case of the clyndrical plasma are shown in section 8 . Section 9 is devoted to the summary.

\section{Linearized MHD Operator}

We consider torus plasma configurations. The stationary ideal MHD equilibria satisfy

$$
\begin{gathered}
\boldsymbol{J}_{0} \times \boldsymbol{B}_{0}=\nabla p_{0}, \\
\nabla \times \boldsymbol{B}_{0}=J_{0}, \\
\nabla \cdot \boldsymbol{B}_{0}=0,
\end{gathered}
$$

where $\boldsymbol{J}_{0}, \boldsymbol{B}_{0}$ and $p_{0}$ are the current density, the magnetic field and the pressure in the equilibrium, respectively. The rationalized Gaussian unit is here used. Taking the scalar product of eq. (1) with $\boldsymbol{B}_{0}$ and $\boldsymbol{J}_{0}$, respectively, we derive

$$
\boldsymbol{B}_{0} \cdot \nabla p_{0}=0, \quad J_{0} \cdot \nabla p_{0}=0 .
$$

Equation (4) shows that the magnetic lines of force and the current lie on a surface $p=$ const. in the equilibrium. A line of force occupies an entire toroidal surface which is called a magnetic surface. We deal with the case that there exists a magnetic axis and around the magnetic axis there exist the magnetic surfaces which do not intersect each other.

Let $\xi$ be the plasma displacement. When we assume the time dependence $\boldsymbol{\xi}(\boldsymbol{r}, t)=\boldsymbol{\xi}(\boldsymbol{r}) \exp (-i \omega t)$, the ideal linearized MHD equations are written as

$$
-\lambda \rho_{0} \xi=F[\xi],
$$

with

$$
\begin{aligned}
\boldsymbol{F}[\boldsymbol{\xi}]= & \left(\nabla\left(\boldsymbol{\xi} \cdot \nabla p_{0}+\gamma p_{0} \nabla \cdot \boldsymbol{\xi}\right)\right. \\
& \left.+\nabla \times \boldsymbol{Q}(\boldsymbol{\xi}) \times \boldsymbol{B}_{0}+\boldsymbol{J}_{0} \times \boldsymbol{Q}(\boldsymbol{\xi})\right\},
\end{aligned}
$$

where $\lambda=\omega^{2}, \boldsymbol{Q}(\boldsymbol{\xi})=\nabla \times\left(\boldsymbol{\xi} \times \boldsymbol{B}_{0}\right)$ and $\rho_{0}$ is the mass density. In general $\rho_{0}$ may be a function of $r$. In the following, we assume that $\rho_{0}$ is a positive constant ( $\rho_{0}=$ 1) for simplicity. Since we assume that the plasma is bounded by a conducting shell, the boundary condition at the plasma surface is expressed by

$$
\xi \cdot n=0,
$$

where $n$ denotes the vector normal to the plasma surface. The eigenvalue problem (5) can be formulated in the weak form as

$$
W[\xi, \eta]=\lambda K[\xi, \eta], \text { for any } \eta,
$$

where

$$
\begin{gathered}
W[\xi, \eta] \equiv \int\left\{\boldsymbol{Q}(\xi) \cdot Q(\boldsymbol{\eta})+J_{0} \times \boldsymbol{\eta} \cdot \boldsymbol{Q}(\xi)\right. \\
\left.+\left(\xi \cdot \nabla p_{0}\right) \nabla \cdot \eta+\gamma p_{0} \nabla \cdot \xi \nabla \cdot \eta\right] \mathrm{d} \tau \\
K[\xi, \eta]=\int \xi \cdot \eta \mathrm{d} \tau
\end{gathered}
$$

with volume element $\mathrm{d} \tau$. From the symmetricity of $W[\xi, \eta]$ we can see that $\lambda$ is real number.

We introduce the coordinate system $(\psi, \theta, \zeta)$ where $\psi$ is a magnetic surface label, while $\theta$ and $\zeta$ are angular coordinates on a toroidal surface. If we deal with 2-D equilibria, we can take $\psi$ as the poloidal magnetic flux within a magnetic surface and $\zeta$ as the toroidal angle $\phi$ which appears in the geometric cylindrical coordinate $(R, \phi, Z)$, while $\theta$ is determined by the condition that the lines of force are straight in the $\theta-\zeta$ plane.

We decompose the displacement vector $\xi$ as

$$
\boldsymbol{\xi}=\xi_{\psi} \frac{\nabla \psi}{|\nabla \psi|^{2}}+\xi_{\mathrm{s}} \frac{\boldsymbol{B} \times \nabla \psi}{B^{2}}+\xi_{\mathrm{b}} \boldsymbol{B} .
$$

Furthermore we expand $\xi_{\psi}, \xi_{s}$ and $\xi_{b}$ into Fourier series with respect to $\theta$ and $\zeta$ 


$$
\begin{aligned}
& \left.\xi_{\psi}=\sum_{m, n} \xi_{\psi}^{m n}(\psi) \exp \mid i(m \theta+n \zeta)\right], \\
& \left.\xi_{\mathrm{s}}=\sum_{m, n} \xi_{\mathrm{s}}^{m n}(\psi) \exp \mid i(m \theta+n \zeta)\right], \\
& \xi_{\mathrm{b}}=\sum_{m, n} \xi_{\mathrm{b}}^{m n}(\psi) \exp [i(m \theta+n \zeta)] .
\end{aligned}
$$

We also define the vectors $\boldsymbol{X}$ and $\boldsymbol{Y}$ as

$$
\boldsymbol{X} \equiv\left(\xi_{\psi}^{m n}\right)^{\mathrm{T}}, \quad \boldsymbol{Y} \equiv\left(\xi_{\mathrm{s}}^{m n}, \xi_{\mathrm{b}}^{m n}\right)^{\mathrm{T}}
$$

$\boldsymbol{X}$ denotes the plasma displacement vectors normal to the magnetic surface and $\boldsymbol{Y}$ in the magnetic surface. When we truncate the Fourier series at $-m_{0} \leq m \leq$ $m_{0}$ and $-n_{0} \leq n \leq 0$, the vectors $\boldsymbol{X}$ and $\boldsymbol{Y}$ have $M_{\mathrm{X}}=$ $2 m_{0} n_{0}+n_{0}+m_{0}+1$ and $M_{Y}=2\left(2 m_{0} n_{0}+n_{0}+m_{0}+1\right)$ components, respectively.

Let $V=\left(H_{0}^{\prime}(0, a ; \mathrm{d} \psi)\right)^{M_{\mathrm{X}}} \times\left(L^{2}(0, a ; \mathrm{d} \psi)\right)^{M_{\mathrm{Y}}}$, where $H^{l}$ denotes the Sobolev space of the order of $l$. We introduce $\boldsymbol{Z} \in V$, and we use the notation $Z=(\boldsymbol{X}, \boldsymbol{Y})$ for an element of $Z$. For any trial function $\boldsymbol{W}=(\boldsymbol{U}, \boldsymbol{V}) \in V$, eq. (8) reduces to the following bilinear form

$$
a[Z, W]=\lambda b[Z, W],
$$

where

$$
a[Z, W]=\int_{0}^{1}\left(U^{\prime H} U^{\mathrm{H}} V^{\mathrm{H}}\right)\left(\begin{array}{lll}
D & C_{1}^{\mathrm{H}} & C_{2}^{\mathrm{H}} \\
C_{1} & A_{11} & A_{21}^{\mathrm{H}} \\
C_{2} & A_{21} & A_{22}
\end{array}\right)\left(\begin{array}{l}
\boldsymbol{X}^{\prime} \\
\boldsymbol{X} \\
\boldsymbol{Y}
\end{array}\right) \mathrm{d} \psi,(
$$

and

$$
b[\boldsymbol{Z}, \boldsymbol{W}]=\int_{0}^{I}\left\{\boldsymbol{U}^{\mathrm{H}} \cdot \boldsymbol{B}_{1} \cdot \boldsymbol{X}+\boldsymbol{V}^{\mathrm{H}} \cdot \boldsymbol{B}_{2} \cdot \boldsymbol{Y}\right\} \mathrm{d} \psi \cdot
$$

Here the prime indicates the derivative with respect to $\psi$ and the superscript $\mathrm{H}$ refers the Hermitian conjugate. $D$, $\boldsymbol{A}_{11}, \boldsymbol{A}_{22}, \boldsymbol{B}_{1}$ and $\boldsymbol{B}_{2}$ are Hermitian matrices. We assume that coefficients in eqs. (15) and (16) are sufficiently smooth. The bilinear form $a[Z, W]$ is continuous and coercive; $b[Z, W]$ is continuous and positive definite. The boundary conditions are given by

$$
X(0)=0, \quad X(1)=0 .
$$

We note that the bilinear form $a[Z, W]$ is related to the Fourier Expanded operator of the lincarized MHD $\hat{F}$ as

$$
a[\mathbf{Z}, \boldsymbol{W}]=b[\hat{F} \mathbf{Z}, \boldsymbol{W}],
$$

\section{Definition of the Good Approximation}

The spectra of the linearized MHD operator $F$ in eq. (6) are to be approximated by means of the finite element method. We here define properties that the approximated spectrum should possess.

Let $\sigma(F) \subset R$ be the spectrum of the operator $F$, and $\sigma\left(F_{\mathrm{h}}\right) \subset \boldsymbol{R}$ the spectrum of the operator $\boldsymbol{F}_{\mathrm{h}}$, where $F_{h}$ is the opcrator approximating $F$. It should be noted that the idcal linearized MHD operator $F$ is noncompact. Therefore, the approximated spectrum $\sigma\left(F_{\mathrm{h}}\right)$ can be polluted, i.e. there exists the series of eigenvatues of $\boldsymbol{F}_{\mathrm{h}}$ which, converges to a value which does not belong to the spectrum of $\boldsymbol{F}$. We say that $\sigma\left(F_{\mathrm{h}}\right)$ is "a good approximation" of $\sigma(\boldsymbol{F})$ when the approximated spectrum $\sigma\left(\boldsymbol{F}_{\mathrm{h}}\right)$ satisfies the following properties to the spectrum $\sigma(F)$;

i) For all $\mu \in \sigma(A)$, there exist the series of eigenvalues of $A_{\mathrm{h}}$ which converges to $\mu$.

ii) There do not exist the series of eigenvalues of $\boldsymbol{F}_{\mathrm{h}}$ which converges to an eigenvalue which does not belong to the spectrum of $\boldsymbol{F}$ (Non-pollution property).

iii) The numerical errors in the eigenvalue scale as $N^{-2\left(p_{2}+1\right)}$, where $N$ is the number of elements and $p_{2}$ refers the degree of base function for the unknown function $Y$. If the coefficients belong to $H^{\alpha}\left(\alpha<2\left(p_{2}+1\right)+1\right)$ but not to $H^{\alpha+1}$, the numerical errors scale as $N^{-(\alpha+1)}$.

\section{Approximation of Function Spaces}

When we apply the finite element method to eq. (14) the mathematical problem is that unknown functions $\boldsymbol{X}$ and $\boldsymbol{Y}$ belong to different function spaces, i.e., there does not exists $Y^{\prime}$ in eq. (14). We here discuss the approximation of function spaces.

The integral region $[0,1]$ is divided into $N$ intervals. There are $N+1$ points $\psi_{0}=0<\psi_{1}<\cdots<\psi_{N}=1$ in $[0,1]$. The $i$-th interval is written as $\left(\psi_{i-1}, \psi_{i}\right)$. For a given integer $p$ we introduce the following finite dimensional function space $K_{\mathrm{h}}^{\mathrm{p}}$;

$$
\begin{gathered}
K_{\mathrm{h}}^{\mathrm{p}}=\{f \mid f \text { is a polynomial of degree } \leq p \\
\text { on } \left.\left(\psi_{i-1}, \psi_{i}\right) \quad i=1,2, \ldots, N\right\} .
\end{gathered}
$$

Furthermore the following two kinds of finite dimensional function spaces $S_{\mathrm{h}}^{p_{1}, c_{1}}$ and $T_{\mathrm{h}}^{p_{2}, c_{2}}$ are defined; For given integers $p_{1}, p_{2}, c_{1}\left(1 \leq c_{1} \leq p_{1}\right)$ and $c_{2}\left(1 \leq c_{2} \leq\right.$ $\left.p_{2}\right)$

$$
\begin{gathered}
S_{\mathrm{h}}^{p_{1}, c_{1}}=\left\{f \mid f \in K_{\mathrm{h}}^{p_{1}} \cap H^{c_{1}}, f(0)=f(1)=0\right\}, \\
T_{\mathrm{h}}^{p_{2}, c_{2}}=\left\{f \mid f \in K_{\mathrm{h}}^{p_{2}} \cap H^{c_{2}}\right\} .
\end{gathered}
$$

Here $c_{2}=0$ denotes that base functions are 
discontinuous on borders of intervals. The dimensions $n_{\mathrm{X}}$ (of the function space $S_{\mathrm{h}}^{p_{1}, c_{1}}$ ) and $n_{\mathrm{Y}}$ (of the function spaces $T_{\mathrm{h}}^{p_{2}, c_{2}}$ ) are given by

$$
\begin{aligned}
n_{\mathrm{X}} & =\left(p_{1}+1\right) N-c_{1}(N-1)-2 \\
& =\left(p_{1}-c_{1}+1\right) N+c_{1}-2, \\
n_{Y} & =\left(p_{2}+1\right) N-c_{2}(N-1) \\
& =\left(p_{2}-c_{2}+1\right) N+c_{2} .
\end{aligned}
$$

We should note that the term -2 in eq. (21) is concerned with the boundary condition.

We apply Ritz-Galerkin method and define the finite element subspace $V_{\mathrm{h}}$ of the function space $V$ as $V_{\mathrm{h}}$ $=\left(S_{\mathrm{h}}^{p_{1}, c_{1}}\right)^{M_{\mathrm{X}}} \times\left(T_{\mathrm{h}}^{p_{2}, c_{2}}\right)^{M_{\mathrm{Y}}}$ i.e. $\boldsymbol{X}$ and $\boldsymbol{Y}$ in eqs. (15) and (16) are expanded with $S_{i} \in S_{\mathrm{h}}^{p_{1, c_{1}}}$ and $T_{i} \in T_{\mathrm{h}}^{p_{2}, c_{2}}$ in the forms

$$
\boldsymbol{X}(\psi)=\sum_{i}^{n_{\chi}} \boldsymbol{X}_{i} S_{i}(\psi), \boldsymbol{Y}(\psi)=\sum_{i}^{n_{Y}} \boldsymbol{Y}_{i} T_{i}(\psi)
$$

We can state the following theorem.

\section{[THEOREM]}

$V_{\mathrm{h}}$ described above is applied for the finite element subspace of the Ritz-Galerkin approximation of the linearized $M H D$ operator. It is assumed that $D$ is positive definite at almost everywhere in $[0,1]$, that det (D) is not zero at almost everywhere in $[0,1]$, and that there exists $\hat{C}_{2}^{\mathrm{H}}$ such that $\hat{C}_{2}^{\mathrm{H}} \cdot C_{2}^{\mathrm{H}}=1$.

The sufficient conditions to obtain the good approximation property are

$$
p_{1} \geq p_{2}+1, c_{1} \leq c_{2}+1,\left(c_{1} \geq 1, p_{2} \geq c_{2} \geq 0\right) .
$$

Base functions which have been used in most of linearized MHD stability codes correspond to the parameters $p_{1}=1, p_{2}=0, c_{1}=1$ and $c_{2}=0$. In [8], it has been pointed out that the combination of the Hermite functions of the third order and of the second order prevents spectrum pollution, i.e. $p_{1}=3, p_{2}=2, c_{1}=3$ and $c_{2}=2$. It has been proved by J. Rappaz in [9] that the sufficient condition for the non-pollution property is $p_{1}=p, p_{2}=p-1, c_{1}=1, c_{2}=0, p \geq 1$. We note that all these conditions satisfy the conditions (24).

\section{Proof of the Theorem for the Good Approximation Property}

In this section we shall prove the theorem described in the previous section.

In section 2 we introduced the eigenvalue problem of MHD equation in the bilinear form eq. (14): $a[Z, W]$ $=\lambda b[\boldsymbol{Z}, \boldsymbol{W}]$. We here consider $\hat{a}[\boldsymbol{Z}, \boldsymbol{W}]=\hat{\lambda} b[\boldsymbol{Z}, \boldsymbol{W}]$ instead of $a[Z, W]=\lambda b[\boldsymbol{Z}, \boldsymbol{W}]$ where $\hat{a}=a+\lambda_{\mathrm{s}} b$ and $\hat{\lambda}=\lambda+\lambda_{\mathrm{s}}$ with a positive constant $\lambda_{\mathrm{s}}$. If the constant $\lambda_{\mathrm{s}}$ is large enough, we can define the linear bounded non-compact operator $\hat{\boldsymbol{F}}_{\lambda_{\mathrm{s}}}{ }^{-1}$ where $\hat{\boldsymbol{F}}_{\lambda_{\mathrm{s}}}=\hat{\boldsymbol{F}}+\lambda_{\mathrm{s}}$. On the other hand, $\hat{a}$ is the positive and continuous bilinear form on $V=\left(H_{n}^{1}(0, a ; \mathrm{d} \psi)\right)^{M_{X}} \times\left(L^{2}(0, a ; \mathrm{d} \psi)\right)^{M_{Y}}$, so that according to the Lax-Milgram theorem, there exists an unique operator $T: V \rightarrow V$ such that

$$
\hat{a}[T Z, W]=b[Z, W], \text { for any } Z, W \in V .
$$

Indecd the operator $T$ accords with the resolvent $\hat{\boldsymbol{F}}_{\lambda_{s}}{ }^{-1}$. In the following, we consider the spectral approximation of $\boldsymbol{T}$.

The linear bounded operator $T$ is the non-compact operator. Therefore, the approximation of $\boldsymbol{T}$ has the difficulty associated with spectral pollution [10]. Descloux-Nassif-Rappaz [11] have verified the mathematical conditions which ensure the efficiency of approximations of the spectrum for a non-compact operator

\section{[THEOREM (Descloux-Nassif-Rappaz)]}

Let $U$ be a Banach space with norm \| \|. Let $A$ be linear bounded operators in $U$. Then sufficient conditions for the good approximation properties are the following two conditions

$$
\begin{aligned}
& P 2: \forall u \in U, \lim _{h \rightarrow 0} \delta\left(u, U_{h}\right)=0, \\
& P 3: \lim _{h \rightarrow 0} \sup _{\substack{u \in U_{h} \\
\|u\|=1}} \delta\left(A u, U_{h}\right)=0 .
\end{aligned}
$$

We note that $P 2$ and $P 3$ are equivalent for a compact operator (sec Appendix I).

We apply this theorem to the operator $T$. Let $V=$ $\left(H_{0}^{1}(0, a ; \mathrm{d} \psi)\right)^{M_{\mathrm{k}}} \times\left(L^{2}(0, a ; \mathrm{d} \psi)\right)^{M_{\mathrm{Y}}}$ and $V_{\mathrm{h}}=\left(S_{\mathrm{h}}^{p_{1}, c_{1}}\right)^{M_{\mathrm{X}}} \times$ $\left(T_{\mathrm{h}}^{p_{2}, c_{2}}\right)^{M_{Y}}$ where $S_{\mathrm{h}}^{\left.p_{1} \cdot c_{1}\right)}$ and $T_{\mathrm{h}}^{p_{2}, r_{2}}$ are the function spaces introduced in the previous section. Then we shall verify that the conditions $P 2$ and $P 3$ are satisfied.

The property $P 2$ is the basic property of the finite elements. We entrust the proof of $P 2$ to textbooks [12]. It remains to verify the $P 3$ property. The detail of the proof of the $P 3$ property is shown in Appendix II. Here only the essence of the proof is shown. The aim is to show that there exist $r \in$

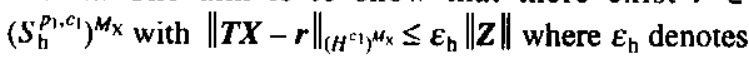
a genetic sequence converging to zero. Since the operator $T$ is the mapping $V \rightarrow V$, under the condition (24), $\boldsymbol{T X}$ can be composed of a function $w \in\left(H_{0}^{c_{1}}\right)^{M_{\mathbf{X}}}$ and a function $\in\left(S_{\mathrm{h}}^{p_{2}+1, c_{2}+1}\right)^{M_{\mathrm{Y}}} \subseteq\left(S_{\mathrm{h}}^{p_{1}, c_{1}}\right)^{M_{\mathrm{Y}}}$. When 
we put $T X=w-G^{-1} \cdot C_{2}^{\mathrm{H}} \cdot \bar{A}_{22}^{-1} \cdot B_{2} \cdot$ and $Y=\phi^{\prime} \in$ $\left(T_{\mathrm{h}}^{p_{2}, c_{2}}\right)^{M_{Y}}$ where $G=D-C_{2}^{H} \cdot A_{22}^{-1} \cdot C_{2}$ and $\bar{A}_{22}=A_{22}$ $+\lambda_{\mathrm{s}} \boldsymbol{B}_{2}$, we can define a compact mapping $V \rightarrow$ $\left(H_{0}^{c_{1}}\right)^{M_{\mathrm{x}}}, \boldsymbol{Z} \rightarrow \boldsymbol{w}$. Hence there exists $p \in\left(S_{\mathrm{h}}^{p_{1}, c_{1}}\right)^{M_{\mathrm{x}}}$ with $\|p-w\|_{\left(H^{\mathrm{c}}\right)^{m_{\mathrm{x}}}} \leq \varepsilon_{\mathrm{h}}\|\mathrm{Z}\|$. Since $S_{\mathrm{h}}^{p_{1}+\mathcal{C}_{1}}$ satisfies the Nitsche-Schatz property (see Appendix I), there exists $q$ $\epsilon\left(S_{\mathrm{h}}^{p_{1}, \mathcal{C}_{1}}\right)^{M_{\mathrm{X}}}$ with $\left\|\boldsymbol{G}^{-1} \cdot \boldsymbol{C}_{2}^{\mathrm{H}} \cdot \overline{\boldsymbol{A}_{22}}{ }^{-1} \cdot \boldsymbol{B}_{2} \cdot \boldsymbol{\phi}-\boldsymbol{q}\right\|_{\left(H^{\boldsymbol{C}^{1}}\right)^{\boldsymbol{M}_{\mathrm{x}}}} \leq$

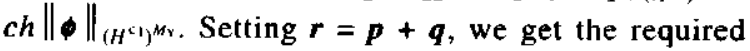
inequality.

\section{Convergence Property}

We here estimate the errors included in the energy integral $a[Z, W]$. It is assumed that the coefficients are sufficiently smooth, i.e., the coefficients belong to $H^{2\left(p_{p}+1\right)+1}$. If the solution $Z=(X, Y)$ is sufficiently smooth, i.e., $\boldsymbol{X} \in H^{p_{1}+1}, \boldsymbol{Y} \in H^{p_{2}}$, Galerkin approximations of the solution $X_{\mathrm{h}} \in S_{\mathrm{h}}^{p_{1}, c_{1}}$ and $Y_{\mathrm{h}} \in T_{\mathrm{h}}^{p_{2}, c_{2}}$ satisfy the following relations

$$
\begin{aligned}
& \left|X-X_{\mathrm{h}}\right|_{1} \leq C N^{-p_{1}}|\boldsymbol{X}|_{p_{1}+1}, \\
& \left|\boldsymbol{Y}-\boldsymbol{Y}_{\mathrm{h}}\right|_{0} \leq C N^{-\rho_{2}+1}|\boldsymbol{Y}|_{p_{2}} .
\end{aligned}
$$

Here $C$ is a constant and \|\|$_{\text {, denotes the Sobolev's }}$ norm of the order of $l$. The errors included in the energy integral $a[Z, W]$ is determined by the order of base functions of $\boldsymbol{Y}$ because of the condition $p_{1} \geq p_{2}+1$. When we select $Z=(X, Y)$ itself as the trial function $W$ $=(\boldsymbol{U}, \boldsymbol{V})$, the errors in the eigenvalues scale as

$$
\left|\mu-\mu^{*}\right| \leq C N^{-2\left(p_{2}+1\right)}
$$

where $\mu$ and $\mu^{*}$ denote the eigenvalue and the approximated onc, respectively.

If the coefficients are not sufficiently smooth, however, the estimation (29) tends to

$$
\left|\mu-\mu^{*}\right| \leq C N^{-(\alpha+1)}
$$

provided that the coefficients belong to $H^{\alpha}\left(\alpha<2\left(p_{2}+\right.\right.$ 1) +1) but not to $H^{\alpha+1}$.

\section{Influence of the Numerical Integration}

The integration in eq. (14) is numerically carried out by using the Gaussian quadrature formula over each element. We shall select $\boldsymbol{Z}$ itself as the trial function $\boldsymbol{W}$. If the polynomials of degree $p$ are employed as the base functions of $\boldsymbol{X}$ in eq. (14) and the condition (24) is satisfied, the highest degree which appears in the integrand in eq. (14) is $2 p$. It is not required that every polynomial which appears be integrated exactly. According to the theory of the finite element method, the condition for the converge of the calculated lowest eigenvalue is that the quadrature formula should be correct at least through degree $2(p-1)$ in the present problem.If we use the $q(>p)$-point quadrature formula, every polynomial must be integrated exactly. Then it is found by the minimax principle that the lowest eigenvalue is approximated from above. But if we employ the just $p$-point quadrature formula, the term of the highest order is not estimated correctly. Although the convergence of the calculated lowest eigenvalue is ensured, the lowest eigenvalue is no longer approximated from above. Whether the eigenvalue is approximated from above or from below will depend on the situation.

It is reported that the lowest eigenvalue is approximated from below in most calculations of ERATO code and TERPSICHORE code [13]. This will be caused by the fact that the quadrature formula which cannol integrate exactly the polynomials is applied in ERATO code and TERPSICHORE code.

\section{Numerical Example}

In order to show the applicability of the present scheme, we applied it to the eigenvalue problem of the cylindrical uniform plasma bounded by a conducting shell. We consider the only one Fourier component with the dependence of $\exp [i(n z+m \theta)]$. When we define $\psi$ $=r^{2} / 2$, eqs. (15) and (16) then reduce to the following quadratic form

$$
a[Z, Z]=\int_{0}^{1}\left(\begin{array}{llll}
X^{\prime} & X & Y_{1} & Y_{2}
\end{array}\right)\left(\begin{array}{cccc}
B^{2}+\gamma_{p} & -\frac{2 B_{\theta}^{2}}{r^{2}} & -\frac{\left(B^{2}+\gamma_{p}\right) G}{B^{2}} & -\gamma_{p F} \\
-\frac{2 B_{\theta}^{2}}{r^{2}} & \frac{F^{2}}{r^{2}}+\frac{2 B_{\theta}^{2}}{r^{3}}-\frac{2 B_{\theta} B_{\theta}^{\prime}}{r^{2}} & -\frac{2 n B_{\theta}}{r} & 0 \\
-\frac{\left(B^{2}+\gamma_{p}\right) G}{B^{2}} & -2 n B_{\theta} & n^{2}+\frac{m^{2}}{r^{2}}+\frac{\gamma p G^{2}}{B^{4}} \frac{\gamma_{p G F}}{B^{2}} \\
-\gamma_{p} F & 0 & \frac{\gamma_{p G F}}{B^{2}} & \gamma p F^{2}
\end{array}\right)\left(\begin{array}{c}
X^{\prime} \\
X \\
Y_{1} \\
Y_{2}
\end{array}\right) \mathrm{d} r,
$$




$$
b[Z, Z]=\int_{0}^{1}\left\{\frac{X^{2}}{r}+r\left(\frac{Y_{1}^{2}}{B^{2}}+B^{2} Y_{2}^{2}\right)\right\} \mathrm{d} r,
$$

where $F=\frac{m B_{\theta}}{r}+n B_{2}, G=\frac{m B_{2}}{r}-n B_{\theta}$.

We shall examine that the coefficients in (31) satisfy the conditions for coefficients in present theorem (see section 4);

$$
D=B^{2}+\gamma_{p}>0 \quad \text { almost everwhere in }[0,1] \text {. }
$$

We determine the equilibrium by giving two profile functions, i.e., the pressure $p(r)$ and the safety factor $q(r)$. Here we consider the case that profile functions are taken as

$$
\begin{gathered}
p(r)=p(0)\left(1-r^{2}\right), \\
q(r)=q(0)(1+c r), c=\frac{q(1)}{q(0)}-1,
\end{gathered}
$$

where the parameters are $p(0)=0.1, q(0)=1 / 6$ and $q(1)=2 / 13$. Hereafter we deal with only the case that $m$ $=2$ and $n=1$. This equilibrium is chosen so that the following three spectra - the Alfvén continuum, the slow continuum, the Sturmian discrete spectrum of fast waves which clusters at the infinity - do not overlap. The schematic picture of these spectra is shown in Fig. 1 [14].

First the non-pollution property of the present method is examined. The number of the point of the Gaussian quadrature formula is fixed for $q=p+1$. When we fix the parameter $p_{1}=2$, we can take the four kinds of combinations of base functions under the condition (24);

$$
\begin{aligned}
& \text { (i) } p_{1}=2, p_{2}=0, c_{1}=1, c_{2}=0, \\
& \text { (ii) } p_{1}=2, p_{2}=1, c_{1}=1, c_{2}=0, \\
& \text { (iii) } p_{1}=2, p_{2}=1, c_{1}=1, c_{2}=1, \\
& \text { (iv) } p_{1}=2, p_{2}=1, c_{1}=2, c_{2}=1 .
\end{aligned}
$$

Figures 2-5 show the numerically calculated spectrum for the cases (i) - (iv) versus the number of intervals. From Figs. 2-5, we can see two eigenvalues of fast waves, as well as the spcctra associated with the continua of the Alfvern waves and the slow waves. Figures show that the spectral pollution docs not occur.

Next, we investigate the convergence property. The results of the numerical convergence are shown in Fig. 6 for the minimum Sturmian discrete eigenvalue. The logarithm of numerical errors versus $\log _{10} N^{-1}$ is

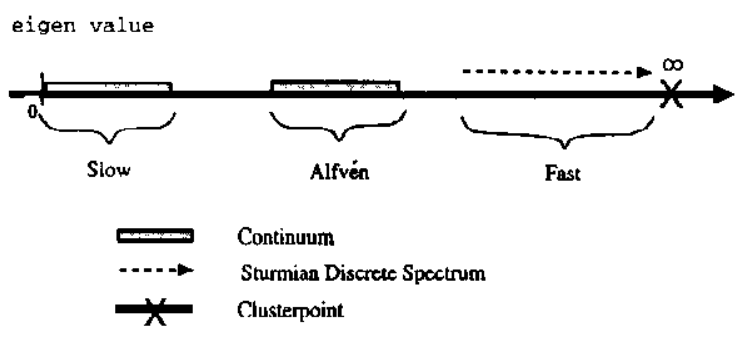

Fig. 1 Schematic picture of the spectrum.

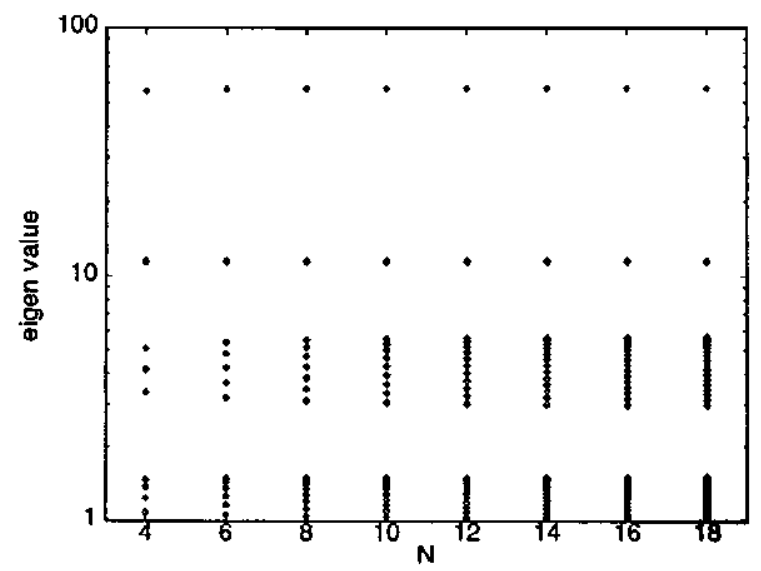

Fig. 2 The numerically calculated spectrum in case of the cylinder model by using the present method as a function of the number of intervals $N$. The parameters are $p_{1}=2, p_{2}=0, c_{1}=1, c_{2}=0$ and $q$ $=3$.

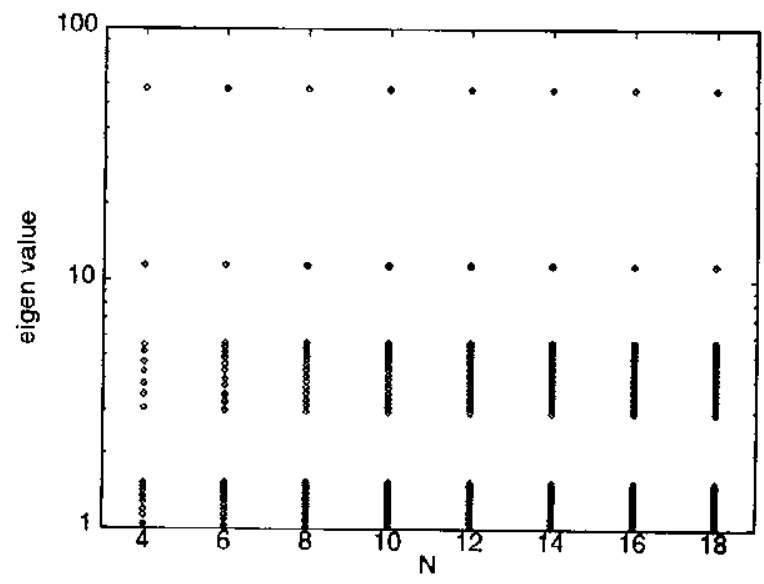

Fig. 3 The numerically calculated spectrum in case of the cylinder model by using the present method as a function of the number of intervals $N$. The parameters are $p_{1}=2, p_{2}=1, c_{1}=1, c_{2}=0$ and $q$ $=3$. 
presented. In Fig. 6(a), the parameters are chosen as $p_{1}=$ $p, p_{2}=p-1, c_{1}=1$ and $c_{2}=0$ for a integer $p(=1,2,3)$. The line with the symbols 0 represents the case $\left(p_{1}=\right.$ $1, p_{2}=0, c_{1}=1, c_{2}=0$ ), while the line with the symbols $\square$ and $\diamond$ represent the case $\left(p_{1}=2, p_{2}=1, c_{1}=1, c_{2}=\right.$ $0)$ and $\left(p_{1}=3, p_{2}=2, c_{1}=1, c_{2}=0\right)$, respectively. The inclinations of these convergence curves are about $2\left(p_{2}\right.$ $+1)$. In Fig. 6(b) the parameters are selected as $p_{1}=p$, $p_{2}=p-1, c_{1}=2$ and $c_{2}=1$. The line with the symbols $O$ represents the case $\left(p_{1}=2, p_{2}=1, c_{1}=2, c_{2}=1\right)$ and the line with the symbols $\square$ represents the case ( $p_{1}=3$, $\left.p_{2}=2, c_{1}=2, c_{2}=1\right)$. The inclinations of these convergence curves are also about $2\left(p_{2}+1\right)$. In both cases the numerical errors are in agreement with analytical one: $\left|\lambda-\lambda^{*}\right|=O\left(N^{-2\left(p_{2}+1\right)}\right)$.

Thirdly, we discuss the influence of the numerical integration. In the equilibrium considered above, the lowest eigenvalue belongs to the continuum, so that it is difficult to discuss about the convergence of the lowest eigenvalue. Hence we consider the other equilibrium characterized by the following profile functions

$$
\begin{aligned}
p(r) & =p(0)\left(1-r^{2}\right)^{2}, \\
q(r) & =q(0) \\
& +r^{2}\left(q(1)-q(0)+\frac{\left(r^{2}-1\right)\left(q^{\prime}(1)-q(1)+q(0)\right)\left(1-y_{\mathrm{s}}\right)}{\left(r^{2}-y_{\mathrm{s}}\right)}\right)
\end{aligned}
$$

where $y_{\mathrm{s}}=\left(q^{\prime}(1)-q(1)+q(0)\right) /\left[q^{\prime}(0)+q^{\prime}(1)-2(q(1)-\right.$ $q(0))]$ and the parameters are $p(0)=0.05, q(0)=0.8$, $q(1)=1.25, q^{\prime}(0)=0.0$ and $q^{\prime}(1)=3.0$. When we select the Fourier modes as $m=1$ and $n=-1$, the internal kink mode becomes unstable. Figure 7 shows the numerically calculated eigenvalue versus $N^{-4}$. The parameters associated with base functions are chosen as $p_{1}=2, p_{2}=$ $1, c_{1}=2$ and $c_{2}=1$. The symbols $\bigcirc$ represent the numerically calculated eigenvalues in the case of using two point quadrature formula, while the symbols $\square$ in the case of using three point formula. We note that in this case the eigenvalue is approximated from below under the influence of the quadrature formula which cannot integrate exactly the polynomials.

\section{Summary}

In this paper we have discussed a scheme to calculate the spectrum of the linearized MHD equation with higher accuracy. In the present method, 2-D (or 3D) stability problem is reduced to $1-\mathrm{D}$ multi-variable

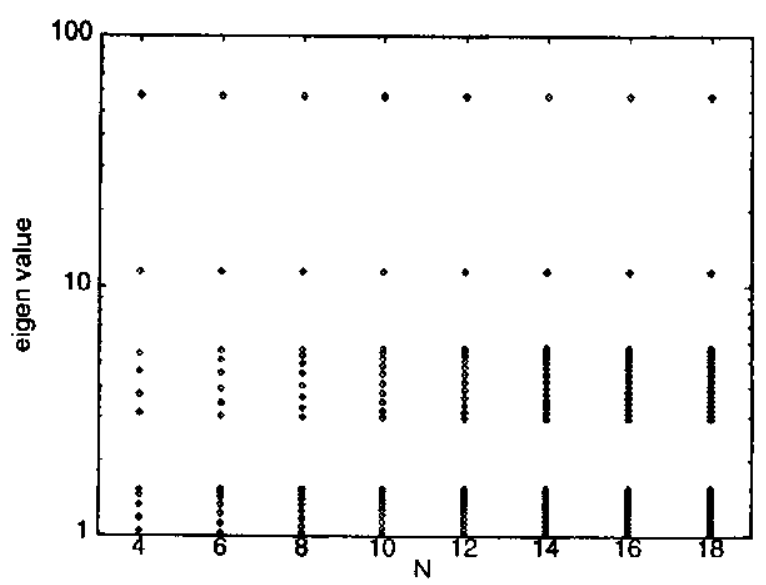

Fig. 4 The numerically calculated spectrum in case of the cylinder model by using the present method as a function of the number of intervals $N$. The parameters are $p_{1}=2, p_{2}=1, c_{1}=1, c_{2}=1$ and $q$ $=3$.

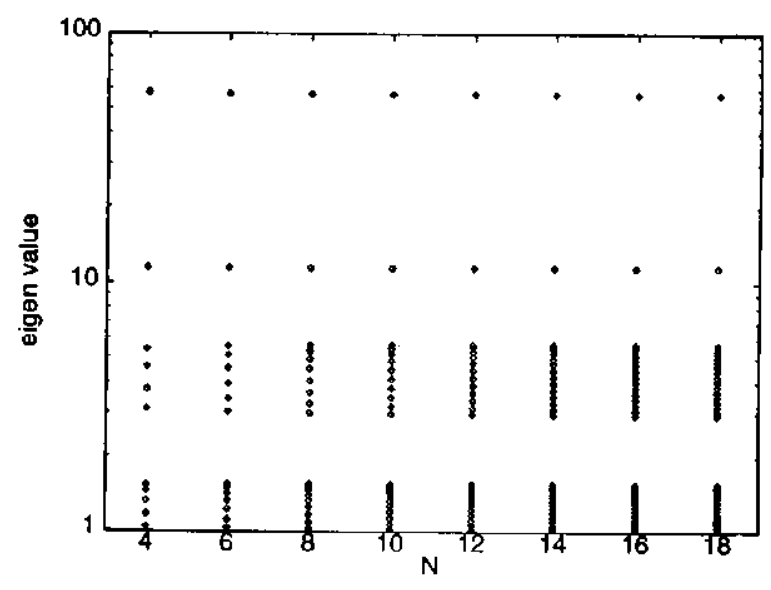

Fig. 5 The numerically calculated spectrum in case of the cylinder model by using the present method as a function of the number of intervals $N$. The parameters are $p_{\uparrow}=2, p_{2}=1, c_{1}=2, c_{2}=1$ and $q$ $=3$.

problem by using the Fourier expansions with respect to poloidal and toroidal angles. Then the finite element method is applied to the minor radius direction. The two kinds of spline functions are employed as the base functions of Ritz-Galerkin approximation; the sufficient conditions (24) for the spline functions to obtain the good approximation property is presented. In most actual calculations the integral cannot be carried out exactly because the equiliblium is given numerically. The present method makes use of the Gaussian 
(a)

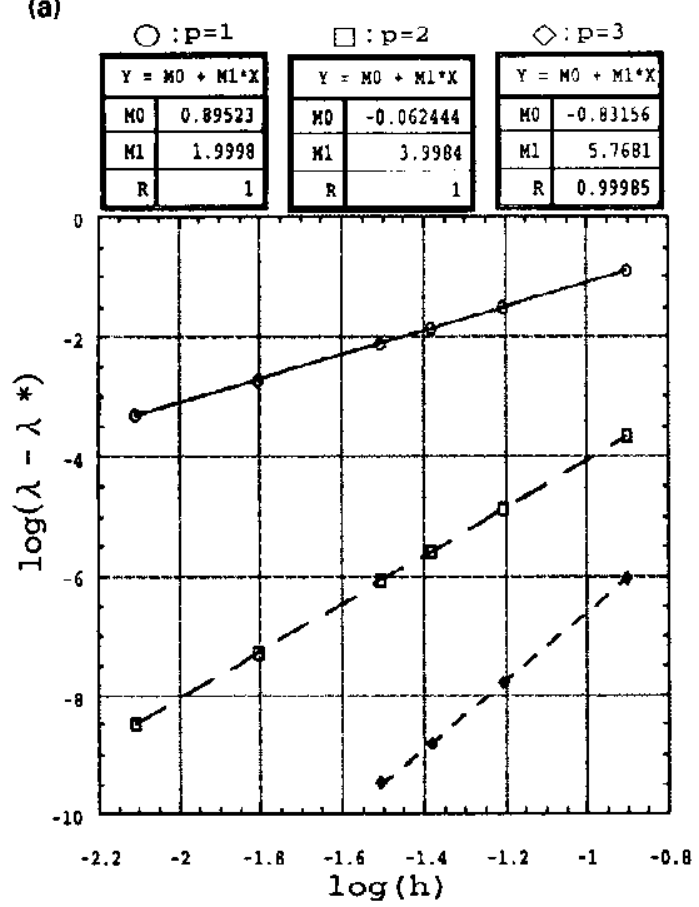

(b)

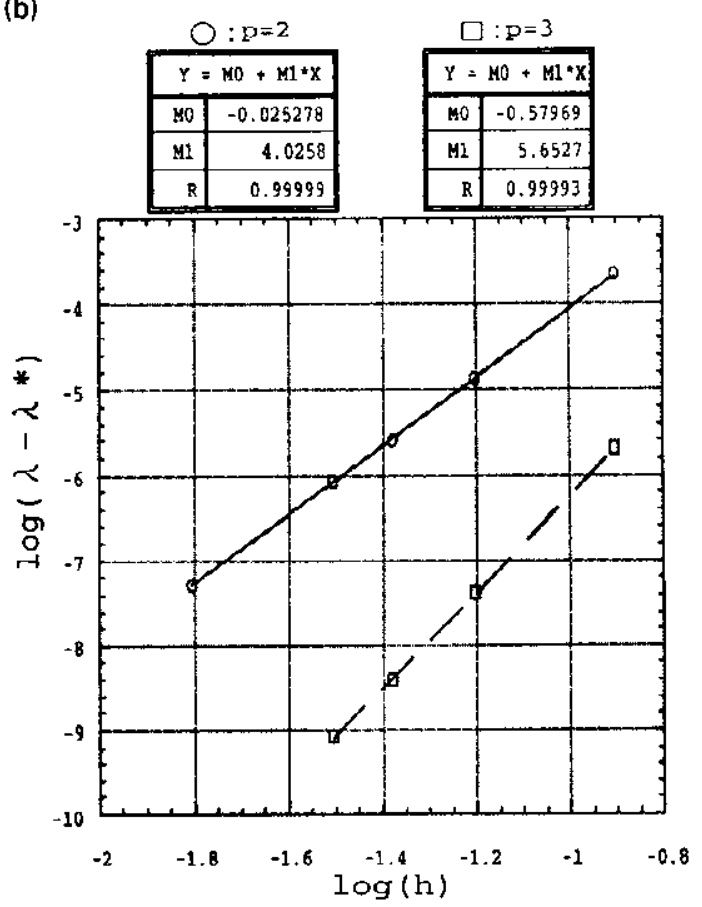

Fig. 6 Numerical convergence for the minimum discrete eigenvalue in the cylinder model. $\ln (a), p_{1}=p, p_{2}=p-1, c_{1}=$ $1, c_{2}=0, q=p+1,\{0: p=1\},\{\square: p=2\},\{0: p=3\}$. In (b), $p_{1}=p, p_{2}=p-1, c_{1}=2, c_{2}=1, q=p+1,\{0: p=2\}$, $\{\square: p=3\}$.

$\lambda \times 10^{-4}$

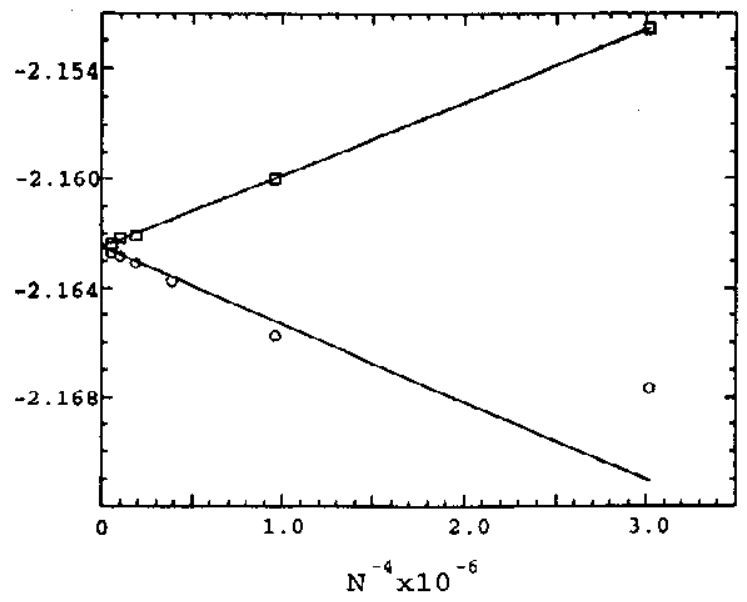

Fig. 7 The numerically calculated eigenvalue of the internal kink mode as a function of the number of intervals $N^{-4}$. The symbols o( I) represent the case of using two (three) point quadrature formula. The parameters associated with base functions are $p_{1}=2, p_{2}=1, c_{1}=2$ and $c_{2}=1$. quadrature formula to evaluate the integral over each elements. The influence of the numerical integration upon the convergence of calculated eigenvalues is discussed.

As an example the present method is applied to the eigenvalue problem of the cylindrical plasma. The good approximation has been obtained, i.e., the spectral pollution is not obscrved and the numerical errors in the discrete eigenvalues are in agreement with analytical one: $\left|\lambda-\lambda^{*}\right|=O\left(N^{-2\left(p_{2}+1\right)}\right)$. The eigenvaluc of the internal kink mode is approximated from below when we use the quadrature formula which cannot integrate exactly the base functions. This is a typical example that the calculated lowest eigenvalues is approximated from below under the influence of the numerical integration.

\section{Acknowledgments}

The authors wish to thank Professor T. Kako, the University of Electro-Communications, for his variable discussions. One of the authors (A. I.) thanks Dr. K. Ichiguchi for his useful discussions. 


\section{Appendix I} article.

We note the theorem and property used in this

[Nitsche-Schatz property] let $\omega$ be a smooth function on a domain $\Omega \subset \boldsymbol{R}^{n}, S_{\mathrm{h}}$ be a family of finite element subspaces of $H^{m}(\Omega)$. Then for $u \in S_{\mathrm{h}}, \inf _{v \in S_{\mathrm{h}}} \| \omega u-$ $v\left\|_{H^{m}} \leq c h\right\| u \|_{H^{m}}$.

[Theorem (Descloux-Nassif-Rappaz)] Let $V$ be $a$ Banach space of norm \|\|$, V_{\mathrm{h}}$ be a sequence of finite dimensional subspace of $V, A$ be a linear bounded operator in $V$. Suppose A compact and $P 2$ verified. Then

$$
\lim _{\mathrm{h} \rightarrow 0} \sup _{\substack{u \in v \\\|v\|=1}} \delta\left(A x, X_{\mathrm{h}}\right)=0,
$$

i.e. for a compact operator P2 and P3 are equivalent.

\section{Appendix II}

As an example Descloux-Nassif-Rappaz have referred to the two-dimensional linearized MHD eigenvalue problem with three components in [11]. We trace the way of the their proof of $P 3$ property in our case.

We consider the region $\Omega=\{\psi \mid \psi \in[0,1] ; \forall v \in$

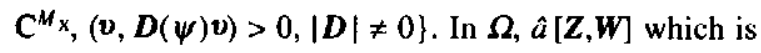
introduced in section 5 is coercive, so that according to the Lax-Milgram theorem, there exists an unique operator $T: V \rightarrow V$ such that

$$
\hat{a}[T Z, W]=b[Z, W], \text { for any } Z, W \in V .
$$

We supposed $V_{\mathrm{h}}=\left(S_{\mathrm{h}}^{p_{1}, c_{1}}\right)^{M_{X}} \times\left(T_{\mathrm{h}}^{p_{2}, c_{2}}\right)^{M_{Y}} \subset\left(H_{0}^{c_{1}}\right)^{M_{\mathrm{x}}} \times$ $\left(H^{L^{2}}\right)^{M_{\mathrm{Y}}}, \boldsymbol{Z} \in V_{\mathrm{h}}, \tilde{\boldsymbol{Z}} \equiv(\tilde{\boldsymbol{X}}, \tilde{\boldsymbol{Y}})=T \boldsymbol{Z}$, i.e.,

$$
\hat{a}[\tilde{\boldsymbol{Z}}, \boldsymbol{W}]=b[\mathbf{Z}, \boldsymbol{W}] \text {, for any } \boldsymbol{W} \in V .
$$

Setting $\boldsymbol{U}=0$ in eq. (37), we obtain

$$
\tilde{\boldsymbol{Y}}=\overrightarrow{\boldsymbol{A}}_{22}^{-1} \cdot\left(\boldsymbol{B}_{2} \cdot \boldsymbol{Y}-\boldsymbol{C}_{2} \cdot \tilde{\boldsymbol{X}}^{\prime}-\boldsymbol{A}_{21} \cdot \tilde{\boldsymbol{X}}\right),
$$

where $\overline{\boldsymbol{A}}_{22}=\boldsymbol{A}_{22}+\lambda_{s} \boldsymbol{B}_{2}$. Substituting this relation for $\tilde{\boldsymbol{Y}}$ into eq. (37) and setting $V=0$, we obtain for any $U \in$ $\left(H_{0}^{\prime}\right)^{M_{\mathbf{y}}}$

$$
\begin{aligned}
& c[\tilde{\boldsymbol{X}}, \boldsymbol{U}]=\int_{\Omega 2}\left\{\boldsymbol{U} \cdot \boldsymbol{B}_{1} \cdot \boldsymbol{X}+\boldsymbol{U} \cdot\left[\boldsymbol{A}_{21}^{\mathrm{H}} \cdot \overline{\boldsymbol{A}}_{22}^{-1} \cdot \boldsymbol{A}_{21}\right.\right. \\
&-\left(\boldsymbol{A}_{11}+\lambda_{5} \boldsymbol{B}_{1}\right)+\boldsymbol{C}_{1}^{\mathrm{H}} \\
&\left.-\left(\boldsymbol{A}_{21}^{\mathrm{H}} \cdot \overline{\boldsymbol{A}}_{22}^{-1} \cdot \boldsymbol{C}_{2}+\boldsymbol{A}_{21}^{\mathrm{H}} \cdot \overline{\boldsymbol{C}}_{2}^{\mathrm{H} \cdot \boldsymbol{G}}\right)^{\mathrm{t}}\right] \cdot \tilde{\boldsymbol{X}} \\
&+\boldsymbol{U}^{\mathrm{H}} \cdot\left[\boldsymbol{C}_{2}^{\mathrm{H}} \cdot \overline{\boldsymbol{A}}_{22}^{-1} \cdot \boldsymbol{A}_{21}-\boldsymbol{A}_{21}^{\mathrm{H}} \cdot \overline{\boldsymbol{A}}_{22}^{-1} \cdot \boldsymbol{C}_{2}\right.
\end{aligned}
$$

$$
\begin{aligned}
& \left.+\boldsymbol{C}_{1}-\boldsymbol{C}_{1}^{\mathrm{H}}-\boldsymbol{A}_{21}^{\mathrm{H}} \cdot \hat{\boldsymbol{C}}_{2}^{\mathrm{H}} \cdot \boldsymbol{G}\right] \cdot \tilde{\boldsymbol{X}} \\
& -\boldsymbol{U}^{\prime} \cdot \boldsymbol{C}_{2}^{\mathrm{H}} \cdot \overline{\boldsymbol{A}}_{22}^{-1} \cdot \boldsymbol{B}_{2} \cdot \boldsymbol{Y} \\
& \left.-\boldsymbol{U} \cdot \boldsymbol{A}_{21}^{\mathrm{H}} \cdot \overline{\boldsymbol{A}}_{22}^{-1} \cdot \boldsymbol{B}_{2} \cdot \boldsymbol{Y}\right) \mathrm{d} \psi
\end{aligned}
$$

with

$$
\begin{aligned}
c & {\left[\phi_{\mathrm{X}}, \phi_{\mathrm{Y}}\right] } \\
& =\int_{\Omega}\left[\phi_{Y}^{\mathrm{H}} \cdot \boldsymbol{G} \cdot \phi_{\mathrm{X}}+\phi_{\mathrm{Y}} \cdot \boldsymbol{A}_{21}^{\mathrm{H}} \cdot \hat{C}_{2}^{\mathrm{H}} \cdot \boldsymbol{G} \cdot \phi_{\mathrm{X}}^{\mathrm{H}} \mathrm{d} \psi\right],
\end{aligned}
$$

where $\hat{C}_{2}^{H}$ is the matrix satisfying $\hat{C}_{2}^{H} \cdot C_{2}^{H}=1$ and $G=$ $\boldsymbol{D}-\boldsymbol{C}_{2}^{\mathrm{H}} \cdot \overline{\boldsymbol{A}}_{22}{ }^{-1} \cdot \boldsymbol{C}_{2}$. Here $\boldsymbol{G}$ is positive definite because $D$ and $\boldsymbol{B}_{2}$ are positive definite and the constant $\lambda_{\mathrm{s}}$ included in $\bar{A}_{22}$ is selected large enough. Hence $c\left[\phi_{\mathrm{X}}, \phi_{\mathrm{Y}}\right]$ is a continuous and coercive bilinear form on $\left(H_{0}^{\mathrm{c}_{1}}\right)^{M_{\mathrm{x}}}$. We here introduce a function $w \in\left(H_{0}^{\mathrm{c}_{1}}\right)^{M_{\mathrm{x}}}$ and a function $\phi \in\left(S_{\mathrm{h}}^{p^{2+1}, c_{2}+1}\right)^{M_{Y}}$. Under the condition (24), $\phi \in\left(S_{\mathrm{h}}^{p_{2}+1, c_{2}+1}\right)^{M_{Y}} \subseteq\left(S_{\mathrm{h}}^{p_{1}, c_{1}}\right)^{M_{Y}}$ and $\phi^{\prime} \in\left(S_{\mathrm{h}}^{p_{2}, c_{2}}\right)^{M_{Y}}$. Substituting $\tilde{X}=\boldsymbol{w}-\boldsymbol{G}^{-1} \cdot \boldsymbol{C}_{2}^{\mathrm{H}} \cdot \overline{\boldsymbol{A}}_{22}^{-1} \cdot \boldsymbol{B}_{2} \cdot \boldsymbol{\phi}$ and $\boldsymbol{Y}=\boldsymbol{\phi}$ $\in\left(r_{\mathrm{h}}^{p_{2}, c_{2}}\right)^{M_{\mathrm{Y}}}$ into eq. (39), we get

$$
\begin{aligned}
& c[\boldsymbol{w}, \boldsymbol{U}]=\int_{\Omega}\left\{\boldsymbol{U} \cdot \boldsymbol{B}_{1} \cdot \boldsymbol{X}+\boldsymbol{U} \cdot\left[\boldsymbol{A}_{21}^{\mathrm{H}} \cdot \overline{\boldsymbol{A}_{22}^{-1}} \cdot \boldsymbol{A}_{21}\right.\right. \\
& -\left(A_{11}+\lambda_{s} B_{1}\right)+C_{1}^{\prime}-\left(A_{21}^{\mathrm{H}} \cdot \bar{A}_{22}^{-1} \cdot C_{2}\right)^{\prime} \\
& \left.-\left(A_{21}^{\mathrm{H}} \cdot \hat{C}_{2}^{\mathrm{H}} \cdot G\right)^{\prime}\right] \cdot\left(\boldsymbol{w}-G^{-1} \cdot C_{2}^{\mathrm{H}} \cdot \bar{A}_{22}^{-1} \cdot \boldsymbol{B}_{2} \cdot \phi\right) \\
& +U^{\prime} \cdot\left[C_{2}^{\mathrm{H}} \cdot \overline{A_{22}^{-1}} \cdot A_{21}-A_{21}^{\mathrm{H}} \cdot \bar{A}_{22}^{-1} \cdot C_{2}+C_{1}-C_{1}^{\mathrm{H}}\right. \\
& \left.-A_{21}^{\mathrm{H}} \cdot \hat{\boldsymbol{C}}_{2}^{\mathrm{H}} \cdot G\right] \cdot\left(\boldsymbol{w}-\boldsymbol{G}^{-1} \cdot \boldsymbol{C}_{2}^{\mathrm{H}} \cdot{\overline{A_{22}}}^{-1} \cdot \boldsymbol{B}_{2} \cdot \boldsymbol{\phi}\right) \\
& -\boldsymbol{U}^{\prime} \cdot \boldsymbol{G} \cdot\left(\boldsymbol{G}^{-1} \cdot \boldsymbol{C}_{2}^{\mathrm{H}} \cdot \bar{A}_{22}^{-1} \cdot \boldsymbol{B}_{2}\right)^{\prime} \cdot \phi \\
& \left.-\boldsymbol{U} \cdot \boldsymbol{A}_{21}^{\mathrm{H}} \cdot \hat{\boldsymbol{C}}_{2}^{\mathrm{H}} \cdot \boldsymbol{G} \cdot\left(\boldsymbol{G}^{-1} \cdot \boldsymbol{C}_{2}^{\mathrm{H}} \cdot \overline{\boldsymbol{A}}_{22}^{-1} \cdot \boldsymbol{B}_{2}\right)^{\prime} \cdot \boldsymbol{\phi}\right\} \mathrm{d} \psi,
\end{aligned}
$$

for any $U \in\left(H_{0}^{c_{1}}\right)^{M_{\mathrm{x}}}$. Then the right member depends on $\phi$, but not on $\phi^{\prime}$. We note that the mapping $V \rightarrow\left(H_{0}^{c_{1}}\right)^{M_{\mathbf{x}}}$, $Z \rightarrow w$ is compact, so that there exists $p \in\left(S_{\mathrm{h}}^{p_{1}, c_{1}}\right)^{M_{x}}$ with $\|\boldsymbol{p}-\boldsymbol{w}\|_{\left(H^{\prime}\right)^{M \times}} \leq \varepsilon_{\mathrm{h}}\|\boldsymbol{Z}\|$, where $\varepsilon_{\mathrm{b}}$ denotes a genetic sequence converging to zero. Since $S_{\mathrm{h}}^{p_{1}, c_{1}}$ satisfies the Nitsche-Schatz property (see Appendix I), there exists $\boldsymbol{q} \in\left(S_{\mathrm{h}}^{p_{1}, c_{1}}\right)^{M_{X}}$ with $\left\|\boldsymbol{G}^{-1} \cdot \boldsymbol{C}_{2}^{\mathrm{H}} \cdot \overline{\boldsymbol{A}}_{22}^{-1} \cdot \boldsymbol{B}_{2} \cdot \boldsymbol{\phi}-\boldsymbol{q}\right\|_{\left(H^{c^{1}}\right)^{M_{\mathrm{x}}}} \leq$ ch \|\|$_{\left(H^{c \mid}\right)^{M v}}$; setting $\boldsymbol{r}=\boldsymbol{p}+\boldsymbol{q}$, we have $\|\tilde{\boldsymbol{X}}-\boldsymbol{r}\|_{\left(H^{c}\right)^{\boldsymbol{N x}_{\mathrm{x}}}}$ $\leq \varepsilon_{\mathrm{h}}\|Z\|$. In order to approximate $\tilde{\boldsymbol{Y}}$, we approximates 
$\tilde{\boldsymbol{X}}$ in eq. (38) by $\boldsymbol{r}$. Since $T_{\mathrm{h}}^{p_{2}, c_{2}}$ satisfies the NitscheSchatz property, there exists $s \in\left(T_{\mathrm{h}}^{p_{2} \cdot c_{2}}\right)^{M_{Y}}$ such that $\| \bar{Y}$

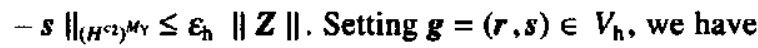
$\|\tilde{Z}-g\| \leq \varepsilon_{\mathrm{h}}\|\boldsymbol{Z}\|$, which proves the $P 1$ property. Finally, we extend the region $\Omega$ to $[0,1]$ by analytic continuation.

\section{References}

[1] R. Gruber, F. Troyon and D. Berger, Comput. Phys. Commun. 21, 323 (1981).

[2] R. Gruber, S. Semenzato, F. Troyon and T. Tsunematsu, Comput. Phys. Commun. 24, 363 (1981).

[3] R.C. Grimm, R.L. Dewar and J. Manickam, J. Comput. Phys. 49, 94 (1983).

[4] D.V. Anderson, W.A. Cooper, R. Gruber, S. Merazzi and U. Schwenn, Int. J. Supercomput. Appl. 4, 3 (1990).

[5] C. Schwab, Phys. Fluids B 5, 9 (1993).
[6] K. Appert, D. Berger, R. Gruber and J. Rappaz, J. Comput. Phys. 18, 284 (1975).

[7] C.Z. Cheng and M.S. Chance, J. Comput. Phys. 71, 124 (1987).

[8] T. Tsunematsu, Handbook of Numerical Calculation (New Version) ed. by Ohno and Isoda, (Ohm Express, Tokyo, 1990) p.434 (in Japanese)

[9] J. Rappaz, Numer. Math. 28, 15 (1977).

[10] K. Appert, D. Berger, R. Gruber and F. Troyon, J. Appl. Math. Phys. (ZAMP) 25, 229 (1974).

[11] J. Descloux, N. Nassif and J. Rappaz, RAIRO Anal. Numér. 12, 97 (1978).

[12] G. Strang and J. Fix, An Analysis of the Finite Element Method (Prentice-Hall, New Jersey, 1973) $\$ 3.2$.

[13] D.V. Anderson, W.A. Cooper, S. Merazzi, U. Schwenn and R. Gruber, Supercomputer 8, 32 (1991).

[14] J.P. Goedbloed, Phys. Fluids 18, 1258 (1975). 